mention unwise when the data are challenged. And, most important, primary data (in whatever form) should be retained for the active life of the research project or for at least five years, unless there is good reason to the contrary.

Such guidelines are sensible, not onerous. Research institutions that have not yet written their equivalents should promptly do so. Many members of the Academy committee David agree. Nevertheless, collectively the group could not see past the fear that guidelines $=$ detailed rules $=$ interference with research freedom. That reflects the notion, no doubt once true, that proper research behaviour is learned by some form of osmosis at the preceptor's knee and that fraud is found out in attempts at replication. The facts now suggest otherwise; whistleblowers are more prominent than replicators, for example.

What has gone wrong? Academy president Frank Press recently noted that, from Archimedes to Bacon to this century (but he may have overlooked the cloud over Ptolemy's calculations), the scientific method has been self-correcting, but is no longer so. "Science is too big, too complex." Indeed, which is why research institutions need to pay attention not only to fraud but also to lesser but more common forms of undesirable behaviour, fraud's precursors. The Academy's report recognizes this but fails to recommend universal remedies beyond education' and local action.

The decline in standards of behaviour in science is most notable at the margins. Always competitive, the endeavour now is more so than ever, sometimes viciously so. Often, competition for has been replaced by competition for money, especially in the biotechnology industry. The idea of a senior scientist working intimately with a handful of students has been relegated to history as laboratories with 50,60 or more members became the norm. Difficult as it may be to accept, scientific etiquette must now be taught. One academy committee member teaching such a course says he begins by telling students that it is not ethical to 'lie' about data, an "idea some of them never thought of".

That is another reason why the report of the academy committee is such a disappointment - it seems unaware of the realities of the laboratory. By all accounts, the group nearly disbanded after its first meeting because many of its members objected to the idea of a study of scientific misconduct on the grounds that it could only make the problem look worse than it is. The same notion is said to have reared its befuddled head when Responsible Science was sent out for peer review. It is, perhaps, remarkable that the report came out at all. But it is ultimately a disservice to perpetuate the idea that the issues of professional behaviour are so complex that even the best minds in science cannot deal with them.

The implementation of procedures for investigating fraud requires judgement and latitude for extenuating circumstances in any given case. It is true that rigid rules about such matters as data retention and sharing reagents would do more harm than good. But the essentials are not complex at all - a truth the research community should be able to accept.

\section{Big Bang brouhaha}

Caution in celebrating last week's announcement of anisotropies in the microwave background would be wise.

If ever a practical demonstration was needed that the whole world is agog to know how the Universe is constructed, last week should have provided it. Hardly a general newspaper in the Western world failed to carry a report on its first page announcing the discovery of a large-scale structure in the pattern of the microwave background radiation on the twodimensional surface of the sky. (Nature's own version, by Professor Joseph Silk, appears on page 741.)

Evidently the microwave satellite COBE is every bit the instrument it was designed to be. But the simple conclusion, that the data so far authenticated are consistent with the doctrine of the Big Bang, has been amplified in newspapers and broadcasts into proof that "we now know" how the Universe began. This is a cause of some alarm.

The search for anisotropies in the microwave background radiation is not a simple matter. People have been looking for variations in the apparent temperature of different patches of the sky of the order of ten millionths of a Kelvin. That they have found them at all is a testament to the power of modern instrumentation. Silk explains how the data gathered have been subjected to the most rigorous checking for consistency, suggesting that the numbers at least can be believed. That the amplitude of the fluctuations observed is consistent with at least some models of galaxy formation is a relief more than a surprise, but the true test will come when other instruments are able to provide measurements of comparable sensitivity on smaller angular scales, where different cosmological theories are more sharply distinguished.

Meanwhile, it is important that the doctrine of the Big Bang is not itself a simple matter. Its origin is supposed to have been the explosion from zero volume at zero time of a corpuscle of energy equivalent to the mass and radiation that now constitute the Universe. Fluctuations have been an intellectual problem for decades; without them, galaxies could not have formed, but there are few physical arguments as to why they should have been present at early times, and even fewer that suggest one spectrum of fluctuations over another. Cosmologists have filled their theoretical universes with some kind of invisible dark matter, to help galaxies form, and many look to inflation - a phase of early exponential - to provide the fluctuations that set the whole process in motion. But for neither dark matter nor inflation is there true independent support, outside the cosmological arena for which they were invented.

Nobody should be surprised, therefore, if the handful of those who reject the Big Bang claim the new data as support for their theories also. It is more relevant that all theories can at last be checked against real data. That will take some time. But it may no longer be the case, as it was some time ago, that Sir Hermann Bondi could begin a public lecture with the observation that "the data in cosmology are so likely to be wrong that I propose to ignore them". 